\title{
Season-aware attraction recommendation method with dual-trust enhancement
}

\author{
Chao Huang ${ }^{\mathrm{a}, *}$, Mengying Liu ${ }^{\mathrm{a}}$, Huiqun Gong ${ }^{\mathrm{b}}$ and Feifei $\mathrm{Xu}^{\mathrm{c}}$ \\ ${ }^{a}$ Department of Management Science and Engineering, School of Economics and Management, Southeast \\ University, Jiangsu, Nanjing 210096, China \\ ${ }^{\mathrm{b}}$ Department of Information Management, School of Economics and Management, Nanjing University of \\ Information Science \& Technology, Jiangsu, Nanjing 210096, China \\ ${ }^{\mathrm{c}}$ Tourism Department, School of Humanities, Southeast University, Jiangsu, Nanjing 210096, China
}

\begin{abstract}
Attraction recommendation plays an essential role in tourism, such as relieving information overload for tourists and increasing sales for tourism operators. When making travel decisions, tourists depend heavily on the personal preferences and suggestions from people they trust. However, most existing attraction recommendation methods focus on the tourist preferences for topics of attractions, yet overlook the seasonality in topic preferences. Additionally, extant studies are generally based on a single type of trust, which may represent trust relations inaccurately. In order to overcome these issues, we propose a novel season-aware attraction recommendation method based on the seasonal topic preferences and dual-trust relations. Firstly, we capture tourists' seasonal topic preferences by analyzing their travel histories along two dimensions: time and attraction. Secondly, we develop a dual-trust relationship (DTR) model based on familiarity-based trust and similarity-based trust, in contrast to existing studies that purely focus on a single type of trust. Thirdly, we propose a novel season-aware attraction recommendation method named SAR-DTR. In a specific season, it predicts ratings based on both topic preferences in the given season and suggestions from tourists they trust. To demonstrate the superiority of the proposed method to other approaches, an empirical study with real-world data was conducted. The experimental results regarding both prediction and recommendation performance are reported.
\end{abstract}

Keywords: attraction recommendation, seasonal topic preference, similarity-based trust, familiarity-based trust

\section{Introduction}

Tourism industry has witnessed an astonishing growth in recent years. According to the report by the World Travel \& Tourism Council (WTTC) in $2016^{1}$, the travel \& tourism directly and indirectly contributed 7.6 trillion Dollar and supported 292 million jobs, separately accounting for $10.2 \%$ of the world's gross domestic product (GDP) and approximately $10 \%$ of all jobs. Apparently, the tourism industry has emerged as a key driver of social and economic development. Both tourism operators and individual tourists have bene-

\footnotetext{
*Corresponding author. Tel.:+86 $138 \quad 1406$ 9012; E-mail: huangchao@seu.edu.cn.

${ }^{1}$ World Travel \& Tourism Council. https://www.wttc.org/research /economic-research/economic-impact-analysis/
}

fited from the boom. However, it also poses unprecedented challenges. For tourism operators, competition is increasingly fierce as many operators have been attracted to this huge and lucrative market. To survive and sustain in this market, travel recommender systems that provide personalized information or products are leveraged as important tools to attract potential tourists and retain existing ones. For tourists, many of them are overwhelmed by the sheer volume of tourism products. Sifting through the enormous amount of travel information is frustrating [1]. Travel recommender systems are frequently employed to relieve information overload and make customized travel plans as they can provide tailored information based on user preferences. Given the critical role of recommender systems in tourism market competition and consumer experi- 
ence, the development of effective travel recommendation methods is highly desirable.

Tourism activity is closely related to tourist preferences. In order to make satisfactory and customized travel recommendations, it is essential to precisely capture tourist preferences [2-3]. In recent years, inferring tourist preferences for topics of attractions from user-generated content has been an emerging issue. Nevertheless, the seasonality in topic preferences, a distinctive feature of tourism, is overlooked by the majority of studies. Tourism is actually a seasonal phenomenon [4]. Tourists' topic preferences are characterized by seasonality, which indicate the dynamic changes of topic preferences with seasons. It is mainly attributed to the distinctive characters of attractions with respect to seasonal context. In addition, tourists' preferences and needs generally change over time, which contribute to variation of travel behaviors as well. Although topic preference has drawn much attention in travel recommendation, research on seasonal topic preference is scarce.

Tourism activity is complex and thus difficult to evaluate before consumption [5-7]. In order to reduce risks and improve the quality of travel experience, tourists heavily depend on the information search when making travel plans. The proliferation of Internet facilitates their access to rich travel information. However, online information can be false, which may lead to misguided travel plans. Therefore, trust has been adopted by recommendation methods to enhance the reliability of recommendation results. Existing trustbased travel recommendation methods are generally based on the trust explicitly issued by tourists or the similarity-based trust [8-10]. Actually, trust inferred from the familiarity between users commonly called familiarity-based trust [11], can potentially be exploited to advance the performance of travel recommendation. It reflects the fact that the tourists take acquaintance as a major information source [12]. Since both similarity-based trust and familiarity-based trust exert considerable influence on travel decision-making process, it would be better to consider these two complementary types of trust when making travel recommendations.

In order to overcome these limitations, we infer the tourists' seasonal topic preferences, develop a dualtrust relationship (DTR) model, and propose a novel season-aware attraction recommendation method. We initially introduce the construction of the proposed DTR model based on familiarity-based trust and similarity-based trust, two essential types of trust for tourists to seek travel suggestions. The familiaritybased trust is computed according to the virtual social interactions, while the similarity-based trust is calculated based on the tourists' seasonal topic preferences, which is acquired by analyzing their own travel histories along two dimensions: time and attraction. Subsequently, we propose a season-aware attraction recommendation method named as SAR-DTR, in a specific season, which generates recommendations based on tourist's personal topic preferences and suggestions from trusted tourists. Finally, using the large scale realworld data, we conduct an empirical study to demonstrate the performance improvement achieved by the proposed method compared with other benchmark approaches.

The remainder of this paper is organized as follows. In section 2, the literature related to our study is reviewed. Section 3 presents the inference of seasonal topic preferences and the DTR model. In section 4, the proposed attraction recommendation method is introduced in detail. The empirical study and results are discussed in section 5, and we conclude the paper with limitations and future studies in section 6 .

\section{Literature review}

Recommender systems can provide personalized recommendations effectively based on user preferences [13-15]. It also reduces information overload and the complexity of information search online [16]. In recent years, due to the fierce competition and information overload in tourism industry, many travel recommender systems have been developed to assist the tourists in making decisions and the tourism operators in attracting tourists [17].

\subsection{Travel recommendation based on tourist preferences}

Tourist preferences are extremely important to making satisfactory travel recommendations [2]. User preferences can be inferred in explicit way and in implicit way [18]. The explicit ways infer user preferences from interactions and feedbacks [19]. Tourists are usually required to provide preferences explicitly. For example, Garcia et al. [3] proposed a travel recommender system that requires tourists to provide general likes and personal data when they register in the system. According to the registration information and historical interactions, the system derives user pref- 
erence model and then generates personalized travel recommendations for the individual or for a group of users. In [20], Vansteenwegen et al. proposed an expert system named City Trip Planner. The City Trip Planner requires tourists to enter the trip constraints and personal interest, and then plans routes for tourists by matching constraints and interests with database of locations. The implicit ways capture user preferences by analyzing user's behaviors [21], such as the analysis of interactions between users and systems [22]. With the proliferation of social media, users generate huge amounts of data online, such as photos, blogs, etc. Inferring preferences from user-contributed data, one of typical explicit ways, has drawn much attention. Besides, because topic is more general and more flexible in describing characters of attractions and tourist preferences, it has been adopted by a growing body of research. For example, Feng et al. [23] inferred the topic distribution vector of user interest by analyzing usercontributed photos and user-generated content. They determined the rank of each product for the user based on topic relevance of user and product. In [24], Jiang et al. extracted topics of user preferences by analyzing the textual description of photos based on the topic model and points of interest were recommended according to the similar users. Xu et al. [25] made use of community-contributed geotagged photos to obtain the topic distribution of user travel histories and then recommended locations to tourists based on collaborative filtering and context constraints.

The literature review suggests that existing studies focus on the topic preferences but overlook the seasonality, a natural character of tourism activity and tourist preferences. To overcome this limitation, we analyze the travel histories in time and attraction to capture tourists' seasonal topic preferences. Given that attractions are the fundamental components of destinations to attract tourists [26-27], we will preliminarily focus on the attraction recommendation in this study.

\subsection{Trust in recommendation}

The boom in social media has significantly changed the domain of information exchange and social media has gained substantial popularity with users to search information. However, users have insufficient information about others, including users with devious intentions, in an online environment [28]. The users have to take risks from false information and fraud when searching information and doing transactions online [29]. In recent years, the recommendation methods have drawn attention to the concept of "trust" that is an essential factor in determining social interactions online and offline, with the objective to reduce risks from trustless users. Trust refers to "a subjective expectation that an agent has about another's future behavior based on the interaction history of their encounters" [30]. In the context of recommendation, trust is defined as "how much users consider the information provided by others relevant with respect to a certain product or preferences" [31-32]. As related studies have pointed out, recommendation methods with trust enhancement can enhance the reliability of recommendation results as well as eliminate the cold-start problem and data sparsity problem [33-34].

Trust-based recommendation methods generally make predictions according to the ratings from trusted users on the target objective weighted by the trust levels [35]. Majority of trust-based recommendation methods utilize trust explicitly issued by users. However, it is time consuming and labor intensive for users to specify trust values [36]. Instead of requiring users to initialize trust values, the trust inference mechanisms utilize user-generated data to infer trust relations, relaxing the users' burden [37-38]. There are many types of trust with respect to its provenance [37]. The similarity between users contributes to the generation of trust, known as similarity-based trust. The rationales behind the similarity-based trust can be explained by homophily theory and social influence theory which indicate that a user tends to connect to the people with similar preferences and that users share similar preferences with people they are connected with, respectively [39-40]. In paper [41], Ziegler et al. proved that there is a strong correlation between trust and interest similarity based on the empirical study. Golbeck [42] demonstrated the correlation between trust and profile similarity by investigating the relationship between similarity in features of profiles and trust. Fernandez-Gago et al. [43] presented a trust model based on the similarity network that is derived from the context similarities among entities. Familiarity is also an essential precondition of trust. The correlational studies suggest that familiarity significantly affects the trust relations between users [44]. Familiarity is frequently adopted to measure the trust levels between users as well. For instance, in [45], Zhang et al. proposed a improved familiarity-based trust model based on the familiarity measurement that takes the prior experience, repeated exposure, level of processing and forgetting rate into consideration. Another related work is [46], which studied the familiarity-based 
trust in the mobile social networks based on the social interactions.

\subsection{Trust-based travel recommendation}

In addition to the personal preferences, tourists also depend on the suggestions from others to make travel plans. Integrating trust into recommendation methods to improve performance has been an emerging issue in tourism. The trust-based travel recommendation methods are mostly based on the trust issued by tourists. According to the trust expressed by users, Avesian et al. [8] proposed a trust-enhanced recommender system named Moleskiing for skiing route recommendation. Moleskiing infers indirect trust values by exploiting trust propagation in the web of trust and then recommends skiing routes based on the user experiences and trust score. In paper [9], García-Crespo et al. proposed a social pervasive recommender system named SPETA. SPETA computes the semantic similarity between the preferences of users and those of his trusted friends, and then evaluates the services based on the collaborative-based filtering method. The similaritybased trust is applied in travel recommendation as well. Hinze et al. [10] proposed the concept of geographic trust that is defined as the trust based on the geographic distance and presented a trust-based recommendation service that considers peer ratings, interpersonal trust and geographical constrains.

One limitation of methods above is that they are purely based on one type of trust, leading to inaccurate description of trust relations between users. Actually, tourists prefer to seek suggestions from people they are familiar with [12]. Thus, it would be reasonable to consider the familiarity-based trust when recommending attractions. To our best knowledge, travel recommendation methods haven't paid attention to the trust model that considers both the similarity-based trust and familiarity-based trust. Therefore, in this study, we construct a dual-trust model with the objective to provide strong support for proposed trust-based attraction recommendation method. Similarity-based trust and familiarity-based trust are comprehensively considered in the construction of the proposed trust model owing to their significant impact on the travel decisions. We further develop a personalized season-aware attraction recommendation method, in a specific season, which predicts ratings based on tourist's topic preferences and trust relations.

\section{Dual-trust relationship model}

This section elaborates on the construction of the proposed DTR model. DTR is a novel trust inference model that comprehensively considers the familiaritybased trust and similarity-based trust, two influential factors on seeking advice. According to DTR, more reliable and more relevant tourists can be selected to provide travel suggestions. Specifically, the construction of DTR is comprised of three phrases. In the first phrase, the degree of familiarity between tourists is calculated based on the social interactions, and the familiarity-based trust value is then computed according to the familiarity. In the second phrase, the seasonal topic preferences are derived from travel histories and the similarity-based trust value is calculated. In the third phrase, we detail the construction of DTR model based on the familiarity-based and similaritybased trust.

\subsection{Familiarity-based trust model}

Familiarity-based trust refers to the trust based on the personal familiarity [11]. Studies indicate that social interactions usually contribute to the familiarity between users [47]. With the proliferation of online social media, users can also share information online through various virtual interactions, such as following others, visiting homepages, commenting on the content, leaving message, thumbs up, and so on, leading to higher familiarity between them. The "following others", "visiting homepage" and "commenting on the content", three primary types of virtual social interactions, have been adopted by numerous social medias. The familiarity in this paper depends on these three types of interactions, with the objective of making the proposed trust model work with majority of social medias and simultaneously reveal the familiarity between users precisely. Suppose two tourists $u_{1}$ and $u_{2}$ in the social media $S M$, each of three kinds of virtual interactions and its value is described as follows.

1. Following others: Following others is a fundamental functionality provided by most social medias. This functionality makes it easy for tourists to get the latest information from people they are interested in. In general, if $u_{1}$ followed $u_{2}$, we refer to $u_{1}$ as follower and $u_{2}$ as followee. Because the information on followee will be automatically pushed by social media services to his followers, the followee is repeatedly exposed to followers, resulting in the familiarity to him 
[47]. Denote the "following others" as $s_{u_{1}, u_{2}}^{f}$. In this study, $s_{u_{1}, u_{2}}^{f}$ is defined as Eq.(1):

$$
s_{u_{1}, u_{2}}^{f}= \begin{cases}1, & u_{1} \text { followed } u_{2} \\ 0, & \text { otherwise }\end{cases}
$$

2. Visiting homepage: Visiting homepage offers opportunity for tourists to gather information from others' homepages to support their travel decisions. The homepage is a webpage where tourists publish content (e.g., blogs and pictures). Let $s_{u_{1}, u_{2}}^{v}$ denotes the "visiting homepage" and it is defined as Eq.(2):

$$
s_{u_{1}, u_{2}}^{v}= \begin{cases}1, & u_{1} \text { visited homepage of } u_{2} \\ 0, & \text { otherwise }\end{cases}
$$

3. Commenting on the content: Tourists can comment on the contents that are published by others to share opinions or seek advice. Denote the "commenting on the content" as $s_{u_{1}, u_{2}}^{c} \cdot s_{u_{1}, u_{2}}^{c}$ is defined as Eq.(3):

$$
s_{u_{1}, u_{2}}^{c}= \begin{cases}1, & u_{1} \text { commented on the content } \\ & \text { published by } u_{2} \\ 0, & \text { otherwise }\end{cases}
$$

Based on the description of social interactions, the degree of familiarity from $u_{1}$ to $u_{2}$, denoted by $f\left(u_{1}, u_{2}\right)$ can be calculated by aggregating three types of interactions, namely $s_{u_{1}, u_{2}}^{f}, s_{u_{1}, u_{2}}^{v}$ and $s_{u_{1}, u_{2}}^{c}$. The $f\left(u_{1}, u_{2}\right)$ is formulated as Eq.(4):

$$
f\left(u_{1}, u_{2}\right)=s_{u_{1}, u_{2}}^{f}+s_{u_{1}, u_{2}}^{v}+s_{u_{1}, u_{2}}^{c}
$$

where $s_{u_{1}, u_{2}}^{f} \in\{0,1\}, s_{u_{1}, u_{2}}^{v} \in\{0,1\}, s_{u_{1}, u_{2}}^{c} \in$ $\{0,1\}$. The more types of social interactions between tourists indicate the higher level of familiarity between tourists. $f\left(u_{1}, u_{2}\right)$ indicates how familiar $u_{1}$ is with $u_{2}$.

Inspired by the positive correlation between familiarity and trust [44], we define the familiarity-based trust from $u_{1}$ to $u_{2}, F T\left(u_{1}, u_{2}\right)$, as Eq.(5):

$$
F T\left(u_{1}, u_{2}\right)=\frac{f\left(u_{1}, u_{2}\right)}{\max \left(f\left(u_{1}, \widetilde{u}\right)\right)}
$$

where $\widetilde{u} \in S M$. As indicated in Equation (4) and (5), the familiarity-based trust is positively associated with social interactions. Without loss of generality, the more familiar tourists are, the higher trust levels they have. Hence, $F T\left(u_{1}, u_{2}\right)$ implies how much $u_{1}$ considers $u_{2}$ trustworthy based on the previous social interactions.

\subsection{Similarity-based trust model}

Similarity-based trust refers to the trust that is generated according to the degree of similarity between users, which is generally the similarity in preferences [41-42]. As already stated, due to the seasonality in attractions, the tourist's topic preferences differ with respect to seasonal context. In this study, the concept of "seasonal topic preferences" is adopted to indicate the tourist preferences for topics of attractions in different seasons. We define the similarity-based trust as the trust based on the similarity in tourists' seasonal topic preferences. Rather than using the questionnaire survey, we capture seasonal topic preferences by analyzing travel histories that are published on social medias by tourists themselves. In this paper, the travel histories in particular refer to a set of visit records, where a visit record indicates that the tourist visited a specific attraction in a certain month.

Denote the season set as $S=\{$ spring, summer, autumn,winter $\}$ and the topic space as $T S=$ $\left\{t_{1}, t_{2}, \ldots, t_{n}\right\}$, where $t_{1}, t_{2}, \ldots, t_{n}$ represent totally different topics. For a specific tourist $u$ in social media $S M$, denote his travel histories as $H_{u}=$ $\left\{h_{j}\right\}, j=1,2, \ldots, m$. A visit record in $H_{u}$ is denoted by $h=(t, a)$, which refers that tourist $u$ visited attraction $a$ in month $t$. To infer the seasonal topic preferences of $u$, each visit record in travel histories is analyzed along travel time (i.e., month) dimension and attraction dimension. Two dimensions are mapped into seasons, namely "spring", "summer", "autumn" and "winter", and topic space $T S$, respectively. To map the travel time into seasons, a year is separated into four seasons by the meteorological division method. Consequently, the method groups March, April and May into spring, June, July and August into summer, September, October and November into autumn, December, January and February into winter. To map attractions into topic space, the topics of attractions that are defined with a unified classification standard are obtained. Then, for a specific tourist $u$, his seasonal topic preferences are inferred as follows.

Firstly, for each visit record $h=(t, a)$ in travel histories $H_{u}=\left\{h_{j}\right\}, j=1,2, \ldots, m$, based on the season determination, the month $t$ can be mapped into season $S_{i}$; according to the topics of attraction $a$ and 
topic space $T S$, attraction $a$ can be mapped into topic space, as a result of which, attraction $a$ is represented as a $n$-dimensional topic vector, denoted by $V_{a}$. The elements in $V_{a}$ are $0 / 1$. That is, if attraction $a$ is featured with topic $t_{i}, t_{i} \in T S$, the $i$ th element in $V_{a}$ is 1 , otherwise 0 . Now, the travel histories can be denoted as $H_{u}^{\prime}=\left\{h_{j}^{\prime}\right\}, j=1,2, \ldots, m$, where $h_{j}^{\prime}=\left(s_{i}, V_{a}\right)$.

Secondly, according to the season $s$ in season set $S$, sum up the topic vectors of attractions that $u$ visited in season $s$. We will get a $4 \times n$ matrix, where the elements are denoted as $p_{u}^{i, j}, i=1,2,3,4 ; j=$ $1,2, \ldots, n$.

Finally, the seasonal topic preferences matrix can be constructed by normalizing the elements $p_{u}^{i, j}$ in the constructed matrix into $[0,1]$, as shown as Eq.(6):

$$
p_{u}^{i, j}=\frac{p_{u}^{i, j}}{\sum_{j=1}^{n} p_{u}^{i, j}}
$$

where $n$ denotes the number of topics in topic space.

Denote the seasonal topic preferences matrix of tourist $u$ as $S T P_{u}$, which presents the preferences of tourist $u$ for topics with respect to the seasonal context. $S T P_{u}^{i}$, the $i$ th row of $S T P_{u}$, is a $n$-dimensional vector and denotes the topic preferences of tourist $u$ in season $S_{i}$ and the element $p_{u}^{i, j}, i=1,2,3,4 ; j=1,2, \ldots, n$ represents the preference of $u$ for topic $t_{j}, t_{j} \in T S$ in season $S_{i}$.

For example, suppose the travel histories of tourist $u$ is $H_{u}=\left\{\left(\right.\right.$ April,$\left.a_{1}\right),\left(\right.$ April,$\left.a_{2}\right),\left(\right.$ May,$\left.a_{3}\right),($ May, $\left.a_{4}\right),\left(\right.$ July,$\left.a_{5}\right),\left(\right.$ July,$\left.a_{6}\right),\left(\right.$ July,$\left.a_{7}\right),\left(\right.$ July,$\left.a_{8}\right),($ October, $\left.a_{9}\right),\left(\right.$ October,$\left.\left.a_{10}\right)\right\}$ and the corresponding topics of attractions in $H_{u}$ are $t_{1}, t_{2}, t_{3}, t_{3}, t_{2}, t_{4}, t_{4}$, $t_{4}, t_{2}, t_{4}$, then the topic space is $T S=\left\{t_{1}, t_{2}, t_{3}, t_{4}\right\}$. The seasonal topic preferences matrix of $u$, denoted as $S T P_{u}$, is shown as follows:

$$
S T P_{u}=\left[\begin{array}{cccc}
0.25 & 0.25 & 0.5 & 0 \\
0 & 0.25 & 0 & 0.75 \\
0 & 0.5 & 0 & 0.5 \\
0 & 0 & 0 & 0
\end{array}\right]_{4 \times 4}
$$

$S T P_{u}$ is a $4 \times 4$ matrix and the element $S T P_{u}^{i j}$ represents the preference of $u$ for topic $t_{j}, t_{j} \in T S$ in season $S_{i}$. Take topic preferences in spring, namely $S T P_{u}^{1}$, as an example. The preferences for topics in topic space (i.e., $t_{1}, t_{2}, t_{3}, t_{4}$ ) are $0.25,0.25,0.5,0$ and $u$ prefers topic $t_{3}$ above all the other topics in spring.
Inspired by the positive correlation between similarity and trust [41-42] and based on the tourists' seasonal topic preferences, the similarity-based trust is defined as the relevance of their preferences. Given the seasonal topic preferences matrices of two tourists $u_{1}$ and $u_{2}$, denoted as $S T P_{u_{1}}$ and $S T P_{u_{2}}$, the similaritybased trust value, $S T\left(u_{1}, u_{2}\right)$, is formulated as Eq.(7):

$$
S T\left(u_{1}, u_{2}\right)=\frac{\sum_{i=1}^{4} \sum_{j=1}^{n} p_{u_{1}}^{i, j} \times p_{u_{2}}^{i, j}}{\sqrt{\sum_{i=1}^{4} \sum_{j=1}^{n}\left(p_{u_{1}}^{i, j}\right)^{2}} \sqrt{\sum_{i=1}^{4} \sum_{j=1}^{n}\left(p_{u_{2}}^{i, j}\right)^{2}}}
$$

where the $p_{u_{1}}^{i, j}$ and $p_{u_{2}}^{i, j}$ are the elements in $S T P_{u_{1}}$ and $S T P_{u_{2}}$, respectively, indicating the preferences of $u_{1}$ and $u_{2}$ for topic $t_{j}, t_{j} \in T S$ in season $S_{i} ; S T\left(u_{1}, u_{2}\right)$ indicates how trustworthy $u_{1}$ considers the information provided by $u_{2}$ to be in terms of similarity in preferences.

\subsection{DTR trust model}

DTR aims at measuring the credibility of tourists in acting as the information source of attraction recommendation. Therefore, the DTR should firstly ensure the reliability of tourists, and then evaluate the relevance of information provided by them. Although the familiarity-based trust and similarity-based trust play significant role in seeking travel advice, they are different in reference significance. Specifically, the proposed similarity-based trust is derived from usercontributed data (i.e., the travel histories), the authenticity of which is the prerequisite for the evaluation of similarity-based trust. In reality, the large amount of false information online poses a great challenge for the proposed similarity-based trust. In comparison, the familiarity-based trust fundamentally depends on the social interactions that are initialized by tourists themselves, which makes familiarity-based trust independent of false information. To ensure the reliability of information from others and enhance the reliability of recommendation results, we will first consider the familiarity-based trust when constructing DTR model. Furthermore, the acquaintances may differ in preferences and suggestions from acquaintances with different preferences are irrelevant. Thus, we further consider the similarity-based trust between tourists with familiarity-based trust relations.

Based on the analysis above, we take the familiaritybased trust as foundation of DTR and further dif- 
ferentiate the familiarity-based trust according to the similarity-based trust. The DTR trust value, denoted by $T\left(u_{1}, u_{2}\right)$, is computed by incorporating the similaritybased trust into familiarity-based trust, as shown in Eq.(8):

$T\left(u_{1}, u_{2}\right)= \begin{cases}0, & F T\left(u_{1}, u_{2}\right)=0 \\ \frac{F T\left(u_{1}, u_{2}\right)+S T\left(u_{1}, u_{2}\right)}{2}, & \text { otherwise }\end{cases}$

where $T\left(u_{1}, u_{2}\right)$ indicates the DTR trust level from $u_{1}$ to $u_{2} ; F T\left(u_{1}, u_{2}\right)$ and $S T\left(u_{1}, u_{2}\right)$ denote the familiarity-based trust value and similarity-based trust value, which are respectively based on the social interactions and seasonal topic preferences.

According to the DTR values, a weighted trust network representing the trust relations between tourists can be constructed, where the weights indicate trustworthiness of tourists. The constructed trust network will be utilized to support the proposed trust-based attraction recommendation method.

\section{Season-aware attraction recommendation method}

Tourists usually make travel plans based on both personal preferences and evaluations (ratings) from trusted tourists to improve the tour quality and reduce potential risks [48-50]. Nevertheless, attractions that are aligned with tourist preferences may be scored lowly by others, and on the contrary, attractions scored highly by others may not cater well to tourist's needs. Therefore, an effective attraction recommendation method should consider both tourist preferences and ratings from trusted tourists. In this section, we propose a novel season-aware attraction recommendation method, which predicts tourists' ratings on attractions based on their topic preferences in the given season and ratings from trusted tourists, who are selected from the constructed trust network according to the DTR values. The proposed method is named as SAR-DTR.

Specifically, with the objective to predict rating $\widehat{R}_{s}\left(u_{0}, a_{0}\right)$ of tourist $u_{0}$ on attraction $a_{0}$ in season $s$, the rating based on preferences, denoted as $\widehat{R}_{s}^{p}\left(u_{0}, a_{0}\right)$, and rating based on trust relations, denoted as $\widehat{R}_{t}\left(u_{0}, a_{0}\right)$, are computed respectively. First, studies indicate that users with similar preferences in the past are more likely to be interested in the same items in the future [51]. Because tourism is featured with seasonality, we predict the tourists' ratings in a specific season according to their similarities in the given season, which is different from existing methods that predict ratings based on the similarities without considering seasonality. Thus, the preferencebased rating $\widehat{R}_{s}^{p}\left(u_{0}, a_{0}\right)$ is calculated according to the tourists who share the similar topic preferences with $u_{0}$ in season $s$. Then, according to the transitivity property of trust, the tourists who are indirectly connected with trust relations are also trustworthy, making more reliable information available for trust-based recommendation methods [52-53]. Thus, we compute $\widehat{R}_{t}\left(u_{0}, a_{0}\right)$ according to multiple levels of tourists with the objective of reducing the negative effects of information deficiency and uncertainty on recommendation methods. Finally, in season $s$, the predicted rating based on both preferences and trust relations, i.e., $\widehat{R}_{s}\left(u_{0}, a_{0}\right)$, is defined on the consideration of $\widehat{R}_{s}^{p}\left(u_{0}, a_{0}\right)$ and $\widehat{R}_{t}\left(u_{0}, a_{0}\right)$ under a turnable parameter $\beta$. The following section describes the proposed recommendation method in detail. The notations used in the method are listed in Table1.

In order to predict preference-based ratings according to the tourists with similar preferences, it is important to accurately identify the similar tourists among all tourists in the travel recommender systems. As aforementioned, the topic preferences are obviously featured with seasonality, rendering it better to take seasonal context into consideration when identifying similar tourists. In section 3, we construct the seasonal topic preferences matrix for each tourist, where each row represents the tourist preferences for topics in a specific season. Let $s$ denote season and $S T P_{u_{0}}^{s}$ and $S T P_{u}^{s}$ represent the topic preferences of tourist $u_{0}$ and $u$ in season $s$, which are $n$-dimensional vectors. The preference similarity of tourist $u_{0}$ and $u$ in season $s$ can be estimated by computing the relevance of their topic preferences vectors $S T P_{u_{0}}^{s}$ and $S T P_{u}^{s}$, which is defined as Eq.(9):

$$
\operatorname{sim}_{s}\left(u_{0}, u\right)=\frac{S T P_{u_{0}}^{s} \times S T P_{u}^{s}}{\left\|S T P_{u_{0}}^{s}\right\| \cdot\left\|S T P_{u}^{s}\right\|}
$$

The higher the $\operatorname{sim}_{s}\left(u_{0}, u\right)$ is, the more similar of $u_{0}$ and $u$ are in preferences in season $s$. Note that because of the seasonality in topic preferences, the similarities between tourists change with seasonal context. According to the similarities between tourists, the top$n$ most similar tourists can be identified, denoted by $U_{u_{0}}^{p}$. In this paper, we set $n$ to 5 . 
Table 1

Notations used in recommendation equations

\begin{tabular}{ll}
\hline Notation & Description \\
\hline$u_{0}$ & The target tourist \\
$a_{0}$ & The target attraction \\
$\widehat{R}_{s}^{p}(u, a)$ & The predicted rating of tourist $u$ on attraction $a$ in season $s$ based on seasonal topic preferences \\
$\operatorname{sim} m_{s}\left(u_{0}, u\right)$ & The preference similarity of $u_{0}$ and $u$ in season $s$ \\
$U_{u}^{p}$ & The neighbors of tourist $u$ selected according to the similarity in preferences \\
$U_{u}^{t}$ & The neighbors of tourist $u$ selected according to the trust relations \\
$N_{k}$ & The set of tourists in $k t h$-level \\
$t_{u}, u$ & Trust value from $u_{0}$ to $u$ \\
$\operatorname{sim}(a, b)$ & The topic similarity of attraction $a$ and $b$ \\
$\widehat{R}_{t}(u, a)$ & The predicted rating of tourist $u$ on attraction $a$ based on trust relations \\
$\widehat{R}_{s}(u, a)$ & The predicted rating of tourist $u$ on attraction $a$ in season $s$ based on preferences and trust relations \\
$\beta$ & A turnable parameter utilized to balance the preference-based rating and trust-based rating \\
\hline
\end{tabular}

Utilizing preference similarity between target tourist $u_{0}$ and other similar tourists in $U_{u_{0}}^{p}$, we predict the preference-based rating of tourist $u_{0}$ on attraction $a_{0}$ in season $s$ as Eq.(10):

$\widehat{R}_{s}^{p}\left(u_{0}, a_{0}\right)=\bar{R}_{u_{0}}+\frac{\sum_{u \in U_{u_{0}}^{p}} \operatorname{sim}_{s}\left(u_{0}, u\right)\left(R_{u, a_{0}}-\bar{R}_{u}\right)}{\sum_{u \in U_{u_{0}}^{p}} \operatorname{sim}_{s}\left(u_{0}, u\right)}$

$$
\begin{aligned}
\bar{R}_{u_{0}} & =\frac{\sum_{a \in A_{u_{0}}} R_{u_{0}, a}}{\left|A_{u_{0}}\right|} \\
\bar{R}_{u} & =\frac{\sum_{a \in A_{u}} R_{u, a}}{\left|A_{u}\right|}, \forall u \in U_{u_{0}}^{p}
\end{aligned}
$$

where $\bar{R}_{u_{0}}$ and $\bar{R}_{u}$ indicate the average attraction rating of $u_{0}$ and $u$, which are separately computed as Equation (11) and (12). $\widehat{R}_{s}^{p}\left(u_{0}, a_{0}\right)$ refers to the level to which tourist $u_{0}$ likes attraction $a_{0}$ based on how other similar tourists feel about $a_{0}$ weighted by the similarities in season $s$. The higher $\widehat{R}_{s}^{p}\left(u_{0}, a_{0}\right)$ indicates the more preferences of $u_{0}$ for $a_{0}$ in terms of topic preferences in season $s$.

Trust-based rating is generated according to multiple levels of tourists with the objective of eliminating the problems generated from information deficiency as well as increasing the credibility of recommendation results. We regard the tourists who are directly trusted by $u_{0}$ and are selected to make recommendations as first level, the selected tourists directly trusted by tourists in first level as second level, and so on. De- note the tourist in $k t h$ level as $N_{k}$. Note that $k$ may be infinite and for the target tourist $u_{0}$, the reliability of tourists in $N_{k}$ will decrease with the increment of $k$ [54]. Thus, it would be better to set the upper limit of $k$. According to the "three degrees of influence rule" [55], we set the maximum of $k$ to 3. Besides, as tourists differ in trustworthiness, they have different reference significance [35]. Instead of selecting tourists randomly, the tourists with higher trust values should be selected with higher priority. For a specific tourist $u_{0}$, we will identify the top- $n$ most trustworthy tourists as his neighbors, denoted by $U_{u_{0}}^{t}$. The $n$ in this paper is set to 5 .

When generating rating $\widehat{R}_{t}\left(u_{0}, a_{0}\right)$ of tourist $u_{0}$ on attraction $a_{0}$ based on trust relations, the tourists in $U_{u_{0}}^{t}$ will be firstly considered. For tourist $u$ in $U_{u_{0}}^{t}$, if $u$ has scored $a_{0}$, denote the rating as $R_{u, a_{0}} . R_{u, a_{0}}$ will be retained to predict $\widehat{R}_{t}\left(u_{0}, a_{0}\right)$ and we will not further consider the tourists who are trusted by $u$, since $u$ has given the rating explicitly. Tourism is a relatively infrequent activity, which leads to the rating matrix sparse. When the tourist didn't score the target attraction, the average of his ratings on the attractions that has the same topics can be utilized as his rating on target attraction. That is, if $u$ didn't score $a_{0}$, we will adopt the average of his ratings on similar attractions as rating from $u$ on $a_{0}$, denoted by $\widehat{R}\left(u, a_{0}\right)$. Specifically, attractions with same topics are similar in characters to some extent. It is reasonable to assume that ratings on similar attractions reflect the evaluation on the target attraction. The similarity between attractions is computed based on their $n$-dimensional topic vectors, which are generated in section 3 . The similarity between attrac- 
tion $a$ and attraction $b$ is defined as Eq.(13):

$$
\operatorname{sim}(a, b)=\frac{V_{a} \times V_{b}}{\left\|V_{a}\right\| \cdot\left\|V_{b}\right\|}
$$

where $V_{a}$ and $V_{b}$ refer to the topic vectors of $a$ and $b$. According to similarities of attractions, the top- $n$ most similar attractions that $u$ has scored can be identified, denoted as $A_{u}$. We adopt the average ratings from $u$ on similar attractions as his rating on $a_{0}$ :

$$
\widehat{R}\left(u, a_{0}\right)=\frac{\sum_{b \in A_{u}} R_{u, b}}{n}
$$

where $b$ denotes the similar attraction and $R_{u, b}$ denotes the rating on similar attraction by $u$.

Although $\widehat{R}\left(u, a_{0}\right)$ can reflect the evaluation of $u$ on $a_{0}$ to some degree, it would be better to consider an adequate number of ratings to mitigate the impact of noisy information [35]. Besides, to facilitate information collection and information sharing, the homepages of tourists are usually open to others, which foster the flow of information in the entire network. Furthermore, the three degrees of influence rule indicates that tourists who are indirectly connected with $u_{0}$ also exert influence on him [55]. Therefore, if $u$ doesn't score $a_{0}$ and $k$ doesn't reach the maximum, after generating $\widehat{R}\left(u, a_{0}\right)$, we will further consider the neighbors of $u$, namely $U_{u}^{t}$. For the tourists in $U_{u}^{t}$, repeat the above process. This process will continue until $k$ reaches the maximum or all tourists in $k t h$ level scored $a_{0}$. Then, for a specific tourist $u$ in $N_{k}$ who didn't scored $a_{0}$, his final predicted rating is computed as Eq.(15):

$$
R_{u, a_{0}}=\frac{\widehat{R}\left(u, a_{0}\right)+\frac{\sum_{v \in U_{u}^{t}} t_{u, v} R_{v, a_{0}}}{\sum_{v \in U_{u}^{t}} t_{u, v}}}{2}
$$

where $\widehat{R}\left(u, a_{0}\right)$ indicates rating based on his ratings on similar attractions; $\frac{\sum_{v \in U_{u}^{t}} t_{u, v} R_{v, a_{0}}}{\sum_{v \in U_{u}^{t}} t_{u, v}}$ is rating from his neighbors. We take the average of two parts as his final evaluation of $a_{0}$.

According to the ratings from direct neighbors of $u_{0}$ on $a_{0}$, we define the trust-based rating $\widehat{R}_{t}\left(u_{0}, a_{0}\right)$ as Eq.(16):

$$
\widehat{R}_{t}\left(u_{0}, a_{0}\right)=\frac{\sum_{u \in U_{u_{0}}^{t}} t_{u_{0}, u} R_{u, a_{0}}}{\sum_{u \in U_{u_{0}}^{t}} t_{u_{0}, u}}
$$

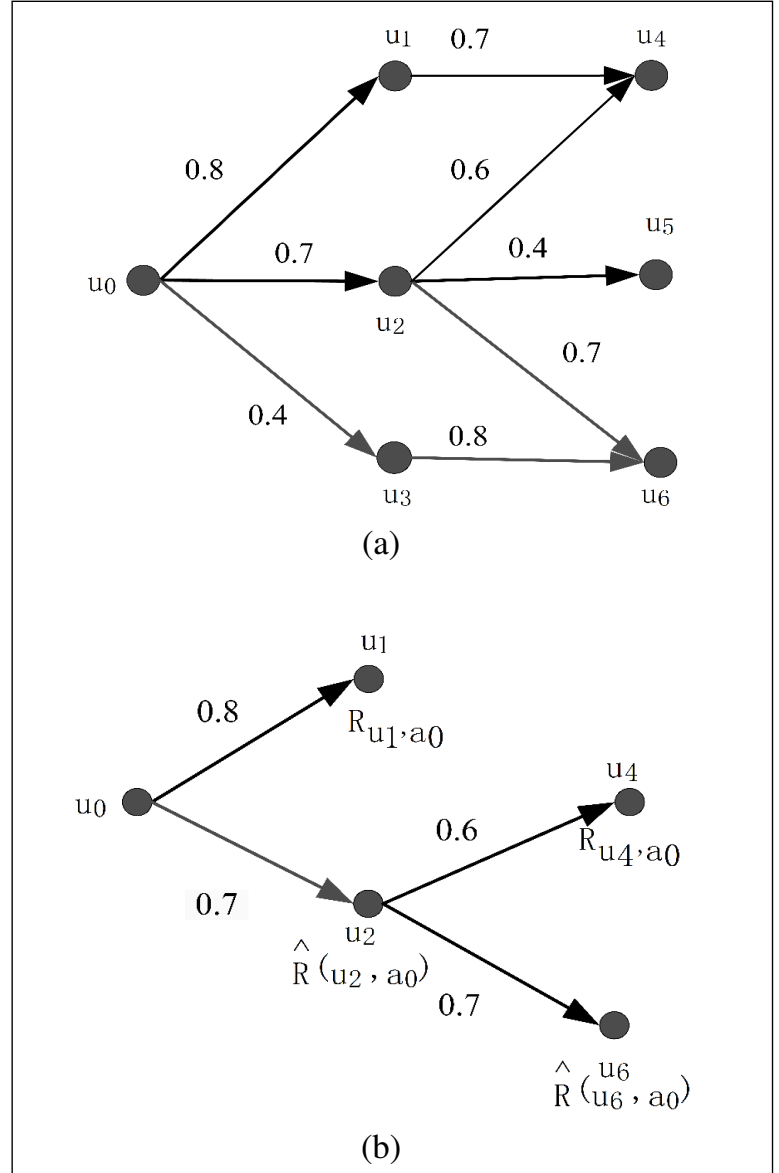

Fig. 1. Example of generating ratings based on trust relations.

where $\widehat{R}_{t}\left(u_{0}, a_{0}\right)$ indicates the $u_{0}$ 's evaluation of $a_{0}$ in terms of trust relations; $t_{u_{0}, u}$ indicates the trust level from $u_{0}$ to $u$.

Fig.1 shows an example to illustrate the proposed method clearly. Fig.1 (a) is a trust network, where the nodes represent the tourists and the weight of each edge denotes the trust level. Suppose the trust-based rating $\widehat{R}_{t}\left(u_{0}, a_{0}\right)$ from $u_{0}$ to attraction $a_{0}$ is to be generated based on trust relations, the maximum of $k$ is 2 , and the top- 2 most trustworthy tourists will be selected as neighbors. Firstly, tourists $u_{1}$ and $u_{2}$ are selected since they are with higher trust levels. Suppose $u_{1}$ scored $a_{0}$ and $u_{2}$ didn't. As for $u_{1}$, his rating on $a_{0}$, i.e., $R_{u_{1}, a_{0}}$, will be retained to generate $\widehat{R}_{t}\left(u_{0}, a_{0}\right)$. On the other hand, because $u_{2}$ doesn't score $a_{0}$, the average rating $\widehat{R}\left(u_{2}, a_{0}\right)$ of his ratings on similar attractions is computed. Now $k$ hasn't reach the maximum and we will further consider tourists who are most trustworthy for $u_{2}$, namely $u_{4}$ and $u_{6}$. We suppose $u_{4}$ scored $a_{0}$ and $u_{6}$ didn't. The rating $R_{u_{4}, a_{0}}$ 
from $u_{4}$ on $a_{0}$ will be retained. As for $u_{6}$, since he didn't score $a_{0}$, the average rating $\widehat{R}\left(u_{6}, a_{0}\right)$ is calculated. Now the $k$ has reach the maximum and the process will stop. Fig.1 (b) shows the ratings that are retained to predict $\widehat{R}_{t}\left(u_{0}, a_{0}\right)$.

Since tourists heavily depend on the personal preferences and suggestions from trusted tourists to make travel decisions, we generate rating according to preference-based rating and trust-based rating with a tunable parameter $\beta$ :

$$
\widehat{R}_{s}\left(u_{0}, a_{0}\right)=\beta \widehat{R}_{s}^{p}\left(u_{0}, a_{0}\right)+(1-\beta) \widehat{R}_{t}\left(u_{0}, a_{0}\right)
$$

where $\widehat{R}_{s}^{p}\left(u_{0}, a_{0}\right)$ denotes the rating based on preferences, $\widehat{R}_{t}\left(u_{0}, a_{0}\right)$ denotes the rating based on trust relations and $\beta \in[0,1]$. In particular, if there is no similar tourist in season $s$ to predict $\widehat{R}_{s}^{p}\left(u_{0}, a_{0}\right)$, $\widehat{R}_{s}\left(u_{0}, a_{0}\right)=\widehat{R}_{t}\left(u_{0}, a_{0}\right)$. If $\widehat{R}_{t}\left(u_{0}, a_{0}\right)$ can't be generated because $u_{0}$ doesn't trust anybody, $\widehat{R}_{s}\left(u_{0}, a_{0}\right)=$ $\widehat{R}_{s}^{p}\left(u_{0}, a_{0}\right)$. Note that although seasonal topic preferences are considered in both preference-based rating and trust-based rating, they play different roles. The seasonal topic preferences in the preference-based rating aim at evaluating the level to which tourists like attractions in terms of their own preferences. In the prediction of trust-based rating, seasonal topic preferences are utilized to evaluate the trustworthiness of information provided by other tourists.

\section{Evaluation and Analysis}

In this section, we conduct an empirical study to evaluate the performance of the proposed attraction recommendation method with large-scale real-world data. Specifically, we first present the data collection and statistical analysis of the dataset in section 5.1. Then, the benchmark methods and the evaluation metrics are introduced in section 5.2. Finally, we detail the experiment results in section 5.3.

\subsection{Experiment setup}

To evaluate our proposed recommendation method with real-world data, we crawled data from Mafengwo (www.mafengwo.cn), a famous online social network that is specialized in tourism in China. Mafengwo provides a platform for tourists to share travel experiences, establish social relations, interact with others online as well as serves as a major information source for tourists to make travel plans. The official data from Mafengwo indicates that 100 million tourists have been attracted to the website, 21 million comments are provided on the website and 382 million travel guides have been downloaded by tourists. Specifically, in Mafengwo, tourists can share travel experiences in the form of articles and pictures, and assign 5-scale integer ratings (from 1 to 5) to express their preferences for attractions. Besides, tourists can follow people they are interested in so that they can get their latest information. The openness of individual homepage makes it easy for tourists to visit others' homepages and comment on the contents freely. Because Mafengwo doesn't provide the topics of attractions, we collect the topics from a famous online tourism operator in China Ctrip (www.ctrip.com). Ctrip plays the leading role in the online travel service and is among the world's top three online travel service operators. To facilitate identification of attractions, Ctirp tags the attractions in a unified standard.

Table 2

Statistics of the experimental dataset

\begin{tabular}{lc} 
Tourists & 6,676 \\
Attractions & 10,827 \\
Ratings & 174,112 \\
Topics & 147 \\
Following others & 394,461 \\
Visiting homepages & 3,582 \\
Commenting on the content & 55,505 \\
Social interactions & 453,548 \\
Average ratings per tourists & 26.08 \\
Average social interactions per tourist & 67.94 \\
Average ratings per attraction & 16.08 \\
\hline
\end{tabular}

We conducted the experiments with attractions in China and tourists who have visited at least one attraction and simultaneously followed more than one tourist in Mafengwo. With the objective to establish the trust network, we initially selected 5 tourists as starting nodes and further moved to the tourists who are followed by the selected nodes. This process continued until there are 6,676 tourists in the network. Subsequently, the tourism data (i.e., travel histories and ratings) and social data (i.e., following others, visiting homepages and commenting on the content) about the tourists in the dataset were collected from Mafengwo. In total, 453,548 social interactions, 10,827 attractions and 174,112 ratings are included in the dataset, where the rating sparsity is $0.2409 \%$. Ultimately, the topics of attractions were collected from Ctrip according to 
the names of attractions. By cleaning up the topics of attractions, 147 topics were selected to build the topic space. The statistics of the experimental dataset are shown in Table 2.

According to the proposed DTR model, the DTR values are calculated based on the social interactions and seasonal topic preferences. In the experiment, 437,966 familiarity-based trust relations are established and the average familiarity-based trust level is 0.636. Besides, 214,705 similarity-based trust relations are established between tourists who are connected with familiarity-based trust relations. Based on the familiarity-based trust and similarity-based trust, we finally establish 437,966 DTR trust relations and the average of DTR values is 0.361 .

\subsection{Recommendation strategies and performance metrics}

In the experiment, we demonstrate the superiority of the proposed SAR-DTR method to the four benchmark methods. Firstly, we select the preference-based method that is based on seasonal topic preferences only (named as STP), to demonstrate the importance of the trust relations in attraction recommendation. Secondly, the trust-based recommendation method that is purely based on dual-trust relations (named as DT) is selected to confirm the necessity of seasonal topic preferences. Finally, two classic collaborative filtering methods, the user-based collaborative filtering method (UCF) and item-based collaborative filtering method (ICF), are selected to demonstrate the superiority of SAR-DTR to the traditional methods and the crucial roles of both seasonal topic preferences and trust relations.

To comprehensively evaluate the prediction performance of our recommendation method, several indicators are used, including Mean Absolute Error (MAE), Root Mean Squared Error (RMSE), Precision, Coverage and F-Measure. Additionally, to evaluate the recommendation performance, the Recommendation Hit Rate (RHR) and Recommendation Loss Rate (RLR) are used.

The prediction accuracy denotes the closeness of predicted rating to the actual rating [54]. The MAE and RMSE, two widely used evaluation metrics in recommender systems, are adopted to measure the prediction accuracy of recommendation methods [13-14]. The smaller the MAE and RMSE are, the more accurately the method predicts. MAE and RMSE are de- fined as Eq. (18) and Eq. (19):

$$
\begin{gathered}
M A E=\frac{\sum_{u, i}\left|R_{u, i}-\widehat{R}_{u, i}\right|}{N} \\
R M S E=\sqrt{\frac{\sum_{u, i}\left(R_{u, i}-\widehat{R}_{u, i}\right)^{2}}{N}}
\end{gathered}
$$

where $R_{u, i}$ denotes the actual rating that tourist $u$ gave to attraction $i ; \widehat{R}_{u, i}$ refers to the predicted rating that is generated by the recommendation method and $N$ indicates the number of tested ratings.

Because of the insufficient information, some ratings can't be predicted. The metric Coverage is applied to measure the ability of recommendation methods in predicting ratings in the context of information insufficiency. The Coverage is defined as the percentage of pair of $\langle$ tourist,attraction $\rangle$, for which the method can predict the ratings [56]. The larger the Coverage is, the more ratings can be predicted. It is represented as Eq. (20):

$$
\text { Coverage }=\frac{P}{T}
$$

where $P$ denotes the number of pairs of 〈tourist, attraction〉 that can be predicted and $T$ indicates the total number of tested ratings.

After converting RMSE into metric Precision, which is in the range of $[0,1]$, we apply F-Measure to combine metrics RMSE and Coverage [56], as Eq. (21) and Eq. (22).

$$
\begin{aligned}
\text { Precision } & =1-\frac{R M S E}{4} \\
F-\text { Measure } & =\frac{2 \times \text { Precision } \times \text { Coverage }}{\text { Precision }+ \text { Coverage }}
\end{aligned}
$$

The higher F-Measure denotes the better performance of recommendation methods with respect to both prediction accuracy and coverage.

An attraction $i$ is recommended to tourist $u$ when the predicted rating $\widehat{R}_{u, i} \geq \delta$, where $\delta$ is the predefined recommendation threshold. The recommendation performance of methods can be evaluated by the metrics RHR and RLR, which are separately defined as follows [57]: 
Table 3

Prediction performance of SAR-DTR with different $\beta$

\begin{tabular}{|c|c|c|c|c|c|c|c|c|c|c|c|}
\hline \multirow{2}{*}{ Season } & \multirow{2}{*}{ Metric } & \multicolumn{9}{|c|}{$\beta$} & \multirow{2}{*}{ Variance } \\
\hline & & 0.1 & 0.2 & 0.3 & 0.4 & 0.5 & 0.6 & 0.7 & 0.8 & 0.9 & \\
\hline \multirow{5}{*}{ Spring } & MAE & 0.4516 & 0.4433 & 0.4380 & 0.4354 & 0.4354 & 0.4376 & 0.4417 & 0.4478 & 0.4555 & 0.00005308 \\
\hline & RMSE & 0.5976 & 0.5869 & 0.5791 & 0.5742 & 0.5724 & 0.5736 & 0.5780 & 0.5853 & 0.5955 & 0.00008827 \\
\hline & Coverage & 0.9705 & 0.9705 & 0.9705 & 0.9705 & 0.9705 & 0.9705 & 0.9705 & 0.9705 & 0.9705 & 0 \\
\hline & Precision & 0.8506 & 0.8533 & 0.8552 & 0.8565 & 0.8569 & 0.8566 & 0.8555 & 0.8537 & 0.8511 & 0.00000555 \\
\hline & F-Measure & 0.9066 & 0.9081 & 0.9092 & 0.9099 & 0.9102 & 0.9100 & 0.9094 & 0.9083 & 0.9069 & 0.00000179 \\
\hline \multirow{5}{*}{ Summer } & MAE & 0.4618 & 0.4556 & 0.4520 & 0.4508 & 0.4516 & 0.4540 & 0.4581 & 0.4644 & 0.4724 & 0.00005164 \\
\hline & RMSE & 0.6031 & 0.5957 & 0.5914 & 0.5902 & 0.5920 & 0.5970 & 0.6049 & 0.6157 & 0.6293 & 0.00016984 \\
\hline & Coverage & 0.9725 & 0.9725 & 0.9725 & 0.9725 & 0.9725 & 0.9725 & 0.9725 & 0.9725 & 0.9725 & 0 \\
\hline & Precision & 0.8492 & 0.8511 & 0.8521 & 0.8525 & 0.8520 & 0.8508 & 0.8488 & 0.8461 & 0.8427 & 0.00001058 \\
\hline & F-Measure & 0.9067 & 0.9077 & 0.9084 & 0.9085 & 0.9083 & 0.9076 & 0.9064 & 0.9049 & 0.9029 & 0.00000352 \\
\hline \multirow{5}{*}{ Autumn } & MAE & 0.4547 & 0.4466 & 0.4405 & 0.4368 & 0.4365 & 0.4390 & 0.4448 & 0.4525 & 0.4620 & 0.00007873 \\
\hline & RMSE & 0.5950 & 0.5831 & 0.5749 & 0.5706 & 0.5704 & 0.5742 & 0.5818 & 0.5933 & 0.6083 & 0.00016811 \\
\hline & Coverage & 0.9772 & 0.9772 & 0.9772 & 0.9772 & 0.9772 & 0.9772 & 0.9772 & 0.9772 & 0.9772 & 0 \\
\hline & Precision & 0.8513 & 0.8542 & 0.8563 & 0.8573 & 0.8574 & 0.8565 & 0.8545 & 0.8517 & 0.8479 & 0.00001049 \\
\hline & F-Measure & 0.9099 & 0.9116 & 0.9128 & 0.9134 & 0.9134 & 0.9129 & 0.9118 & 0.9101 & 0.9080 & 0.00000347 \\
\hline \multirow{5}{*}{ Winter } & MAE & 0.4347 & 0.4278 & 0.4245 & 0.4244 & 0.4271 & 0.4320 & 0.4393 & 0.4490 & 0.4607 & 0.00015234 \\
\hline & RMSE & 0.5749 & 0.5657 & 0.5603 & 0.5587 & 0.5609 & 0.5670 & 0.5767 & 0.5899 & 0.6064 & 0.00025232 \\
\hline & Coverage & 0.9830 & 0.9830 & 0.9830 & 0.9830 & 0.9830 & 0.9830 & 0.9830 & 0.9830 & 0.9830 & 0 \\
\hline & Precision & 0.8563 & 0.8586 & 0.8599 & 0.8603 & 0.8598 & 0.8583 & 0.8558 & 0.8525 & 0.8484 & 0.00001581 \\
\hline & F-Measure & 0.9153 & 0.9166 & 0.9174 & 0.9176 & 0.9173 & 0.9164 & 0.9150 & 0.9131 & 0.9108 & 0.00000518 \\
\hline \multirow{5}{*}{ Overall } & MAE & 0.4507 & 0.4433 & 0.4388 & 0.4369 & 0.4377 & 0.4407 & 0.4460 & 0.4534 & 0.4627 & 0.00007430 \\
\hline & RMSE & 0.5927 & 0.5829 & 0.5764 & 0.5734 & 0.5739 & 0.5780 & 0.5854 & 0.5961 & 0.6099 & 0.00014783 \\
\hline & Coverage & 0.9758 & 0.9758 & 0.9758 & 0.9758 & 0.9758 & 0.9758 & 0.9758 & 0.9758 & 0.9758 & 0 \\
\hline & Precision & 0.8519 & 0.8543 & 0.8559 & 0.8567 & 0.8565 & 0.8556 & 0.8537 & 0.8510 & 0.8475 & 0.00000925 \\
\hline & F-Measure & 0.9096 & 0.9110 & 0.9120 & 0.9124 & 0.9123 & 0.9117 & 0.9107 & 0.9091 & 0.9072 & 0.00000304 \\
\hline
\end{tabular}

$$
\begin{aligned}
& R H R=\frac{|(u, i)| \widehat{R}_{u, i} \geq \delta, R_{u, i} \geq \delta \mid}{|(u, i)| \widehat{R}_{u, i} \geq \delta \mid} \\
& R L R=\frac{|(u, i)| \widehat{R}_{u, i}<\delta, R_{u, i} \geq \delta \mid}{|(u, i)| \widehat{R}_{u, i}<\delta \mid}
\end{aligned}
$$

where $R_{u, i}$ indicates the actual rating that tourist $u$ gave to attraction $i$. The RHR evaluates the probability that the attractions recommended by recommendation method are also scored highly by tourists. The RLR computes the probability that the attractions evaluated highly by tourists are not recommended by the method.

\subsection{Experimental results}

In order to evaluate the performances of recommendation methods, the dataset is divided into two parts according to the time of travel: the travel activities occurred before 2015 as training dataset $(163,734$ ratings), and those occurred in 2015 as evaluation dataset (10,378 ratings). The training dataset is utilized to infer tourists' seasonal topic preferences, construct the DTR trust network and predict ratings for the pair of $\langle$ tourist, attraction $\rangle$ in evaluation dataset. The evaluation dataset is utilized to evaluate the prediction performance and recommendation performance of the methods. Note that the SAR-DTR method and STP method take into consideration of seasonal context, while the other three methods (DT, ICF and UCF) are indifferent to the season. We not only compare their overall performance, but also compare the seasonal performance of SAR-DTR with benchmark methods. The overall performance is computed by averaging the seasonal performances across the four seasons.

We firstly compare the performance of SAR-DTR with different $\beta$. From Equation (17), we can observe that the bigger the $\beta$ is, the more important role the 


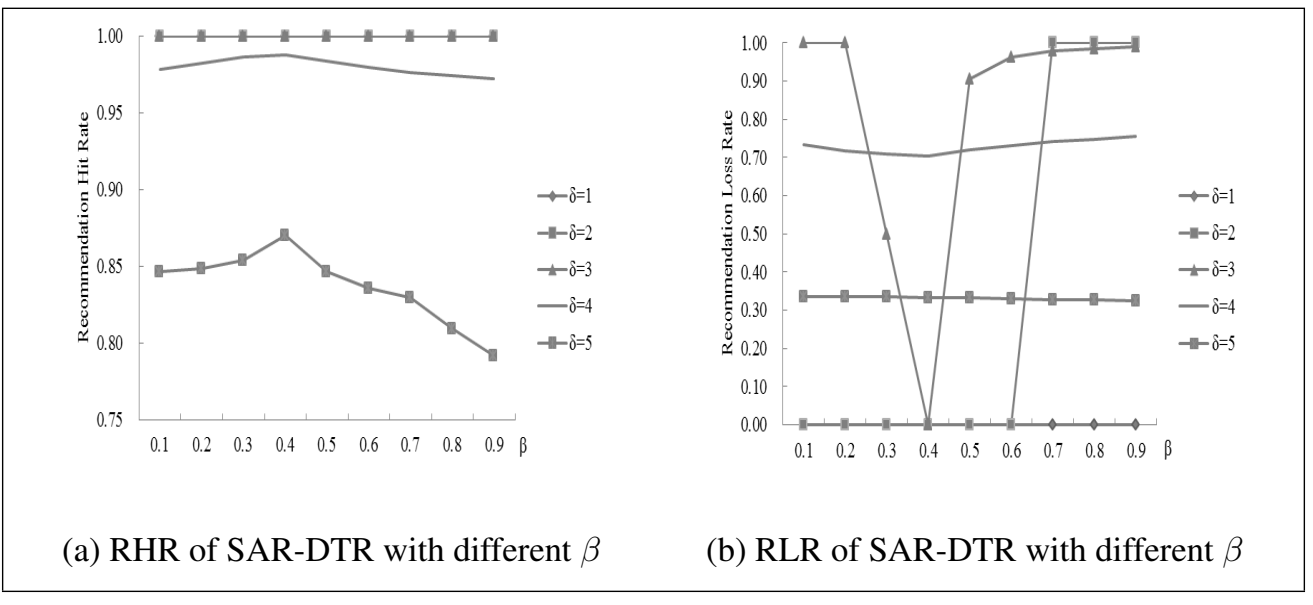

Fig. 2. The overall recommendation performance of SAR-DTR with different $\beta$.

Table 4

Prediction performance comparison between SAR-DTR with benchmark methods

\begin{tabular}{ccccccc}
\hline Method & Season & MAE & RMSE & Coverage & Precision & F-Measure \\
\hline \multirow{3}{*}{ SAR-DTR } & Spring & 0.4354 & 0.5742 & 0.9705 & 0.8565 & 0.9099 \\
& Summer & 0.4508 & 0.5902 & 0.9725 & 0.8525 & 0.9085 \\
& Autumn & 0.4368 & 0.5706 & 0.9772 & 0.8573 & 0.9134 \\
& Winter & 0.4244 & 0.5587 & 0.9830 & 0.8603 & 0.9176 \\
& Overall & 0.4369 & 0.5734 & 0.9758 & 0.8567 & 0.9124 \\
\hline \multirow{3}{*}{ STP } & Spring & 0.5074 & 0.6377 & 0.6120 & 0.8406 & 0.7083 \\
& Summer & 0.5431 & 0.6992 & 0.5971 & 0.8252 & 0.6929 \\
& Autumn & 0.5240 & 0.6644 & 0.6852 & 0.8339 & 0.7523 \\
& Winter & 0.5348 & 0.6802 & 0.7204 & 0.8299 & 0.7713 \\
& Overall & 0.5273 & 0.6704 & 0.6537 & 0.8324 & 0.7312 \\
\hline DT & & 0.4603 & 0.6043 & 0.9745 & 0.8489 & 0.9074 \\
\hline UCF & & 0.6208 & 0.7815 & 0.8379 & 0.8046 & 0.8209 \\
\hline
\end{tabular}

preference-based rating plays in rating prediction. We firstly examine the influence of $\beta$ on prediction performance and recommendation performance of SARDTR. The $\beta \in(0,1)$ and the step is set to 0.1 in this experiment.

Table 3 shows that $\beta$ has a trivial effect on the prediction performance of SAR-DTR. For example, with respect to the overall performance of SAR-DTR with different $\beta$ on MAE and RMSE, the maximum differences are merely 0.0258 and 0.0365 , respectively. In terms of Variance, we can observe that with the change of $\beta$, the variation of the index values are very small and the performance on Coverage doesn't change. The experimental results indicate that $\beta$ has no significant influence on SAR-DTR. To make the proposed SARDTR integrally perform best, we determine $\beta$ according to the overall performance of SAR-DTR on F-
Measure, an aggregate indicator that synthetically considers the prediction accuracy and Coverage. As shown in Table 3, the SAR-DTR obtains the best overall prediction performance on F-Measure when $\beta=0.4$.

The recommender systems recommend attractions to tourists only when the predicted rating is equal to or greater than a predefined level named as recommendation threshold, which is denoted by $\delta$. $\delta$ will influence the recommendation performance and the higher $\delta$ indicates the more strict recommendation condition. Because the rating levels in this experiment are ranged from 1 to 5 , then $\delta \in\{1,2,3,4,5\}$. Fig. 2 depicts the overall recommendation performance of SAR-DTR with different $\beta$ under each $\delta$. As we can see in Fig.2, when $\beta=0.4$, the SAR-DTR generally has the highest RHR and the lowest RLR under every $\delta$. 
Table 5

Recommendation performance comparison between SAR-DTR with benchmark methods

\begin{tabular}{|c|c|c|c|c|c|c|c|c|c|c|c|}
\hline \multirow{2}{*}{ Method } & \multirow{2}{*}{ Season } & \multicolumn{2}{|c|}{$\delta=1$} & \multicolumn{2}{|c|}{$\delta=2$} & \multicolumn{2}{|c|}{$\delta=3$} & \multicolumn{2}{|c|}{$\delta=4$} & \multicolumn{2}{|c|}{$\delta=5$} \\
\hline & & RHR & RLR & RHR & RLR & RHR & RLR & RHR & RLR & RHR & RLR \\
\hline \multirow{5}{*}{ SAR-DTR } & Spring & 1 & 0 & 1 & 0 & 1 & 0 & 0.9917 & 0.7355 & 0.8462 & 0.3367 \\
\hline & Summer & 1 & 0 & 1 & 0 & 1 & 0 & 0.9831 & 0.6441 & 0.8774 & 0.3324 \\
\hline & Autumn & 1 & 0 & 1 & 0 & 1 & 0 & 0.9877 & 0.7398 & 0.8077 & 0.3304 \\
\hline & Winter & 1 & 0 & 1 & 0 & 1 & 0 & 0.9904 & 0.7363 & 0.9036 & 0.3308 \\
\hline & Overall & 1 & 0 & 1 & 0 & 1 & 0 & 0.9882 & 0.7139 & 0.8587 & 0.3326 \\
\hline \multirow{5}{*}{ STP } & Spring & 1 & 1 & 1 & 1 & 1 & 0.9783 & 0.9738 & 0.8163 & 0.7946 & 0.3933 \\
\hline & Summer & 1 & 0 & 1 & 0 & 1 & 1 & 0.9717 & 0.7265 & 0.7044 & 0.4050 \\
\hline & Autumn & 1 & 0 & 1 & 1 & 1 & 1 & 0.9779 & 0.7994 & 0.7258 & 0.3893 \\
\hline & Winter & 1 & 0 & 1 & 1 & 1 & 0.9844 & 0.9645 & 0.8425 & 0.7521 & 0.4044 \\
\hline & Overall & 1 & 0.25 & 1 & 0.75 & 1 & 0.9907 & 0.9720 & 0.7962 & 0.7442 & 0.3980 \\
\hline DT & & 1 & 0 & 1 & 1 & 0.9998 & 1 & 0.9710 & 0.7548 & 0.8571 & 0.3360 \\
\hline $\mathrm{ICF}$ & & 1 & 1 & 1 & 1 & 0.9999 & 0.9976 & 0.8654 & 0.9267 & 0.2875 & 0.3689 \\
\hline UCF & & 1 & 1 & 1 & 1 & 0.9999 & 0.9976 & 0.8956 & 0.9013 & 0.3552 & 0.3664 \\
\hline
\end{tabular}

According to the analysis above, the proposed SARDTR exhibits the best overall prediction performance and recommendation performance when $\beta=0.4$. We will subsequently show the performance comparison between SAR-DTR method and other benchmark methods under $\beta=0.4$. Table 4 compares the prediction performances of different recommendation methods. From Table 4, we can draw several conclusions as follows.

Firstly, under the condition that both SAR-DTR and STP account for the seasonal context, SAR-DTR substantially outperforms STP in each season. In terms of overall performance, the MAE and RMSE of SARDTR are separately 0.0904 and 0.097 lower than those of STP. Meanwhile, SAR-DTR outperforms STP by $49.27 \%$ with respect to Coverage and its Precision and F-measure are respectively $2.92 \%$ and $24.78 \%$ higher than STP as well. The performance advantages of SAR-DTR over STP verify that the consideration of trust relations in attraction recommendation can significantly improve the prediction accuracy and coverage.

Secondly, in each season, SAR-DTR precedes DT, in terms of MAE, RMSE, Precision and F-Measure, under the condition that both methods consider trust relations. As for the overall performance, the MAE and RMSE of SAR-DTR are separately $5.08 \%$ and $5.11 \%$ lower than those of DT. In the meantime, compared with DT, the SAR-DTR has $0.92 \%$ higher Precision and $0.55 \%$ higher F-Measure. Although the Coverage of SAR-DTR in spring and summer are slightly lower than those of DT, its overall performance is superior to DT. The experimental results confirm that the consid- eration of seasonality can increase prediction accuracy without reducing coverage.

Thirdly, compared with the two traditional methods, the performance improvement of SAR-DTR is more significant. With regard to the overall performance comparison between SAR-DTR and ICF, the MAE and RMSE of SAR-DTR are respectively $29.62 \%$ and 26.63\% lower, while its Coverage, Precision and FMeasure are separately $0.1379,0.0521$ and 0.0915 higher. In contrast with UCF, the SAR-DTR has $27.71 \%$ lower MAE and $25.08 \%$ lower RMSE, meanwhile, the performances of SAR-DTR on Coverage, Precision and F-Measure are separately $0.1379,0.0481$ and 0.0894 higher.

Table 5 and Fig. 3 compare the recommendation performance of proposed SAR-DTR method with those of other methods under each recommendation threshold $\delta$, in terms of RHR and RLR. Fig.3 (a) and (b) separately depict the overall performance comparison on RHR and RLR. We can observe that compared with other four benchmark methods, the SAR-DTR has the highest RHR and lowest RLR. That is, the propose SAR-DTR can recommend more satisfactory attractions to tourists and lose less business opportunities. Concretely speaking, comparing SAR-DTR with STP, we can observe that under each $\delta$, SAR-DTR significantly outperforms STP with respect to both RHR and RLR. Table 5 shows that SAR-DTR precedes STP in each season. Besides, averaged across the four seasons, the RHRs and RLRs of SAR-DTR also outperform those of STP under each $\delta$. Even under the strict condition, namely $\delta=5$, the RHR of SAT-DTR 


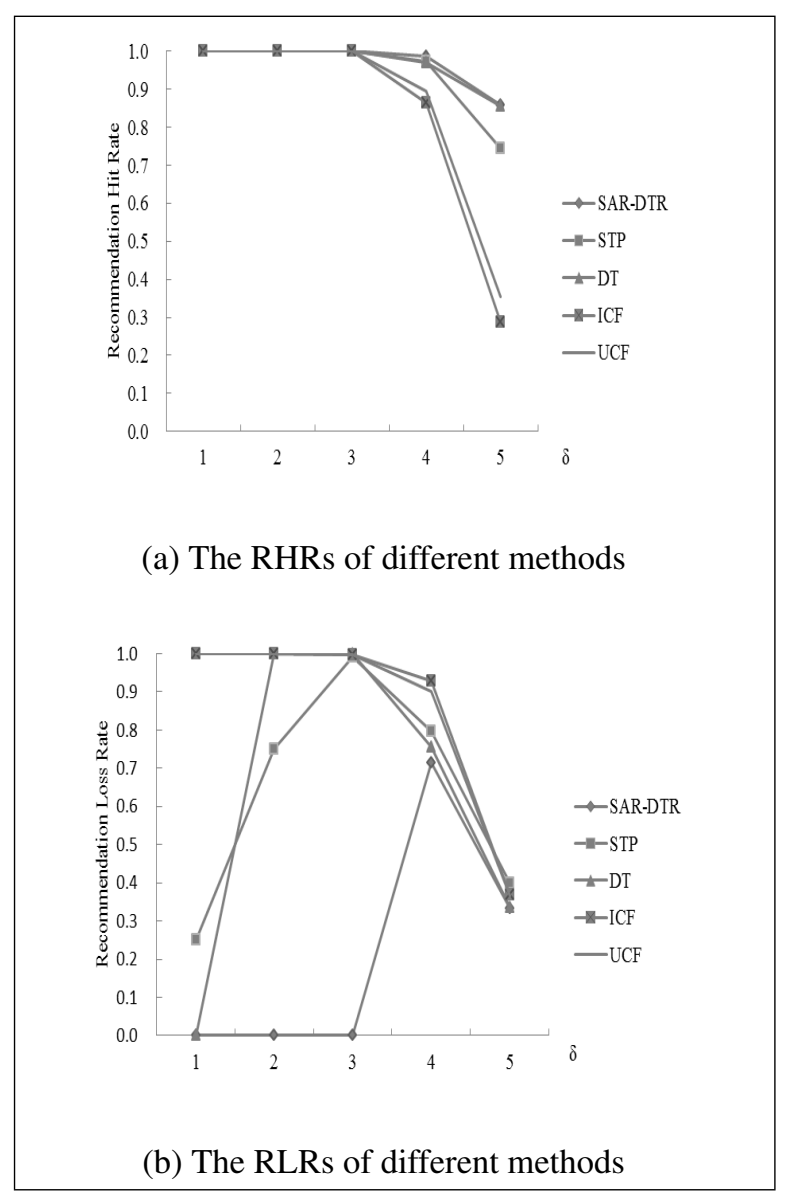

Fig. 3. The overall recommendation performance of different methods.

reaches 0.8587 , which indicates that when the $\delta$ is set to 5 , on average, $85.87 \%$ attractions recommended by SAR-DTR are also scored highly by tourists. In comparison, the RHR of STP is $0.7442,13.33 \%$ lower than that of SAR-DTR. Meanwhile, the RLR of SAR-DTR under $\delta=5$ is 0.0654 lower than that of STP. In other word, 100 attractions recommended by SAR-DTR can lose 6.54 less business opportunities than that recommended by STP. As for the performance comparison between SAR-DTR and DT, the overall performances of SAR-DTR are better than those of DT, despite that in spring and autumn, the RHR of SAR-DTR is lower than that of DT. Given $\delta=4$ as an example. SARDTR outperforms DT on RHR by $1.77 \%$, meanwhile, the RLR of SAR-DTR is $5.42 \%$ lower than that of DT. Compared with ICF and UCF, the performance advancement of SAR-DTR on recommendation performance is more significant. In each season, SAR-DTR is obviously superior to ICF and UCF under each $\delta$, in terms of RHR and RLR. Averaged RHRs and RLRs across four seasons, the overall performances of SARDTR substantially precede ICF and UCF as well. For example, under $\delta=4$, the SAR-DTR outperforms ICF by $14.19 \%$ and UCF by $10.34 \%$ with respect to RHR. In the meantime, the RLR of SAR-DTR is $22.96 \%$ lower than that of ICF and $20.79 \%$ lower than that of UCF.

Because tourism activities are of low frequency, attraction recommendation may suffer severe cold-start problem. We specially compare the recommendation results of cold-start tourists. In this paper, the tourists with less than five visit records are identified as the cold-start tourists [58]. Table 6 shows the overall performance comparison for cold-start tourists. We can observe that SAR-DTR performs well in dealing with cold-start problem. As shown in Table 6, although SAR-DTR has $1.04 \%$ lower Coverage than DT, it outperforms STP that ignores trust relations and DT that overlooks seasonal topic preferences, in terms of all the other metrics. Compared with ICF and UCF, the performance advantages of SAR-DTR on cold-start problem are more outstanding.

\section{Conclusion}

In order to provide effective decision support for tourism operators and tourists, it is highly desirable to develop attraction recommendation methods that jointly consider tourist preferences and trust relations. Existing attraction recommendation methods focus on tourists' topic preferences but overlook the seasonality. On the other hand, they are based on a single type of trust, whereas overlooking the fact that there are multiple types of trust that influence the travel decisionmaking process. Our study addressed these gaps by proposing a season-aware attraction recommendation method with dual-trust enhancement. It contributes to the literature on four aspects. First, we proposed the inference method of seasonal topic preferences, which can capture tourists' topic preferences in a specific season from their travel histories. Second, we constructed the dual-trust relationship (DTR) model that infers the trust relations based on similarity-based trust and familiarity-based trust, in contrast to existing studies that merely focus on a single type of trust. Third, we proposed the season-aware attraction recommendation method (SAR-DTR) that recommends attractions according to the seasonal context, seasonal topic preferences and DTR trust relations. Finally, the em- 
Table 6

Comparison results for cold-strat tourists

\begin{tabular}{|c|c|c|c|c|c|c|c|c|c|c|c|c|c|c|c|}
\hline & \multicolumn{5}{|c|}{ Prediction performance } & \multicolumn{10}{|c|}{ Recommendation performance } \\
\hline & \multirow{2}{*}{ MAE } & \multirow{2}{*}{ RMSE } & \multicolumn{3}{|c|}{ Coverage Precision F-Measure } & \multirow{2}{*}{\multicolumn{2}{|c|}{$\begin{array}{c}\delta=1 \\
\text { RHR RLR }\end{array}$}} & \multirow{2}{*}{\multicolumn{2}{|c|}{$\begin{array}{c}\delta=2 \\
\text { RHR RLR }\end{array}$}} & \multirow{2}{*}{\multicolumn{2}{|c|}{$\begin{array}{c}\delta=3 \\
\text { RHR RLR }\end{array}$}} & \multicolumn{2}{|c|}{$\delta=4$} & \multicolumn{2}{|c|}{$\delta=5$} \\
\hline & & & & & & & & & & & & RHR & RLR & RHR & RLR \\
\hline SAR-DTR & 0.4434 & 0.5771 & 0.8493 & 0.8557 & 0.8521 & 1 & 0 & 1 & 0 & 1 & 0 & 0.9792 & 0.6476 & 0.8500 & 0.2124 \\
\hline STP & 0.6620 & 0.8116 & 0.1687 & 0.7971 & 0.2751 & 1 & 0 & 1 & 0 & 1 & 0 & 0.9550 & 0.6609 & 0.4792 & 0.3394 \\
\hline DT & 0.4563 & 0.5929 & 0.8582 & 0.8518 & 0.8550 & 1 & 0 & 1 & 0 & 1 & 0 & 0.9649 & 0.6788 & 0.7500 & 0.2269 \\
\hline $\mathrm{ICF}$ & 0.8018 & 0.9917 & 0.4253 & 0.7521 & 0.5433 & 1 & 1 & 1 & 1 & 1 & 1 & 0.7444 & 0.9521 & 0.1111 & 0.2540 \\
\hline UCF & 0.8724 & 1.0677 & 0.4253 & 0.7331 & 0.5383 & 1 & 1 & 1 & 1 & 1 & 1 & 0.7681 & 0.9362 & 0.1395 & 0.2585 \\
\hline
\end{tabular}

pirical study was conducted with real-world data to compare the performance of SAR-DTR with benchmark methods. The experimental results demonstrate that the SAR-DTR outperforms the other methods in terms of both prediction performance and recommendation performance and that the comprehensive consideration of seasonal topic preferences and trust relations contributes to the performance improvement of SAR-DTR. The results also demonstrate the performance advantages of SAR-DTR over the benchmark methods with respect to the cold-start tourists. Under the condition of considering seasonality factor, we further incorporate more factors in future studies, such as the space factor and price factor to capture tourist preferences precisely.

\section{Acknowledgments}

This research is supported by the National Natural Science Foundation of China under Grant Nos.71671038 and 41571133.

\section{References}

[1] B. Pan and D. Fesenmaier, A Typology of Tourism-Related Web Sites: Its Theoretical Backgound and Implications, Information Technology \& Tourism 3(3) 2000, 155-166.

[2] Y. Huang and L. Bian, A Bayesian network and analytic hierarchy process based personalized recommendations for tourist attractions over the Internet, Expert Systems with Applications 36(1) (2009), 933-943.

[3] I. Garcia, L. Sebastia and E. Onaindia, On the Design of Individual and Group Recommender Systems for Tourism, Expert Systems with Applications 38 (2011), 7683-7692.

[4] R. Ahas, A. Aasa, Ü. Mark, T. Pae and A. Kull, Seasonal tourism spaces in Estonia: Case study with mobile positioning data, Tourism management 28(3) (2007), 898-910.

[5] M.R. Williams, A history of computing technology, IEEE Computer Society Press, 1997.
[6] S. Klein and H. Werthner, Information technology and tourism a challenging relationship, Springer-Verlag Wien, 1999.

[7] R.C. Lewis and R.E. Chambers, Marketing leadership in hospitality: foundations and practices, John Wiley and Sons, 1999.

[8] P. Avesani, P. Massa and R. Tiella, A trust-enhanced recommender system application: Moleskiing, Proceedings of the 2005 ACM symposium on Applied computing, 2005, 15891593.

[9] A. García-Crespo, J. Chamizo, I. Rivera, M. Mencke, R. Colomo-Palacios and J.M. Gómez-Berbś, SPETA: Social pervasive e-Tourism advisor, Telematics and Informatics 26(3) (2009), 306-315.

[10] A. Hinze and Q. Quan, Trust-and location-based recommendations for tourism, On the Move to Meaningful Internet Systems: OTM 2009, Springer Berlin Heidelberg, 2009, 414-422.

[11] X. Qiao, X. Li, Z. Su and D. Cao, A context-awareness dynamic friend recommendation approach for mobile social network users, International Journal of Advanced Intelligence 3(2) (2011), 155-172.

[12] I. Llodrà-Riera, M.P. Martínez-Ruiz, A.I. Jiménez-Zarco and A. Izquierdo-Yusta, A multidimensional analysis of the information sources construct and its relevance for destination image formation, Tourism management 48 (2015), 319-328.

[13] H. Ma, H. Yang, M.R. Lyu and I. King, Sorec: social recommendation using probabilistic matrix factorization, Proceedings of the 17th ACM conference on Information and knowledge management, 2008, 931-940.

[14] J. Lu, D. Wu, M. Mao, W. Wang and G. Zhang, Recommender system application developments: a survey, Decision Support Systems 74 (2015), 12-32.

[15] W. Wang, G. Zhang and J. Lu, Member contribution-based group recommender system, Decision Support Systems 87 (2016), 80-93.

[16] D.Y. Yeh and C.H. Cheng, Recommendation system for popular tourist attractions in Taiwan using Delphi panel and repertory grid techniques, Tourism Management 46 (2015), 164-176.

[17] M. Batet, A. Moreno, D. Sánchez and V. Aïda, Turist@: Agentbased personalised recommendation of tourist activities, Expert Systems with Applications 39(8) (2012), 7319-7329.

[18] U. Hanani, B. Shapira and P. Shoval, Information filtering: Overview of issues, research and systems, User modeling and user-adapted interaction 11(3) (2001), 203-259.

[19] R. Alton-Scheidl, R. Schmutzer, P.P. Sint and G. Tschertru, Voting and rating in Web4Groups, 1997.

[20] P. Vansteenwegen, W. Souffriau, C.V. Berghe and D.V. Oudheusden, The city trip planner: an expert system for tourists, $E x$ - 
pert Systems with Aplications 38(6) (2011), 6540-6546.

[21] G. Adomavicius and A. Tuzhilin, Toward the next generation of recommender systems: A survey of the state-of-the-art and possible extensions, IEEE transactions on knowledge and data engineering 17(6) (2005), 734-749.

[22] L. Castillo, E. Armengol, E. Onaindía, L. Sebastiá, J. González-Boticario, A. Rodríguez, S. Fernández, J.D. Arias and B. Borrajo, SAMAP: An user-oriented adaptive system for planning tourist visits, Expert Systems with Applications 34(2) (2008), 1318-1332.

[23] H. Feng and X.M. Qian, Mining User-contributed Photos for Personalized Product Recommendation, Neurocomputing 129 (2014), 409-420.

[24] S. Jiang, X. Qian, J. Shen and T. Mei, Travel recommendation via author topic model based collaborative filtering, International Conference on Multimedia Modeling, Springer International Publishing, 2015, 392-402.

[25] Z.X. Xu, L. Chen and G. Chen, Topic based Context-Aware Travel Recommendation Method Exploiting Geotagged Photos, Neurocomputing 155 (2015), 99-107.

[26] N. Leiper, Tourist attraction systems, Annals of tourism research 17(3) (1990), 367-384.

[27] G. Richards, Tourism attraction systems: Exploring cultural behavior, Annals of tourism research 29(4) (2002), 1048-1064.

[28] K. Thirunarayan and P. Anantharam, Trust networks: Interpersonal, sensor, and social, Collaboration Technologies and Systems (CTS), 2011 International Conference on. IEEE, 2011, 1321.

[29] A. J $\phi$ sang, R. Ismail and C. Boyd, A survey of trust and reputation systems for online service provision, Decision support systems 43(2) (2007), 618-644.

[30] L. Mui, M. Mohtashemi and A. Halberstadt, A computational model of trust and reputation, Proceedings of the 35th Annual Hawaii International Conference on. IEEE, 2002, 2431-2439.

[31] D. Ben-Shimon, A. Tsikinovsky, L. Rokach, A. Meisles, G. Shani and L. Naamani, Recommender system from personal social networks, Advances in Intelligent Web Mastering, Springer Berlin Heidelberg, 2007, 47-55.

[32] P. Massa and P. Avesani, Trust-aware recommender systems, Proceedings of the 2007 ACM conference on Recommender systems, 2007, 17-24.

[33] J. O'Donovan and B. Smyth, Trust in recommender systems, Proceedings of the 10th international conference on Intelligent user interfaces, 2005, 167-174

[34] H. Ma, I. King and M.R. Lyu, Learning to recommend with social trust ensemble, Proceedings of the 32 nd international ACM SIGIR conference on Research and development in information retrieval, 2009, 203-210.

[35] S. Deng, L. Huang and G. Xu, Social network-based service recommendation with trust enhancement, Expert Systems with Applications 41(18) (2014), 8075-8084.

[36] X. Li, M. Wang, T.P. Liang, A multi-theoretical kernel-based approach to social network-based recommendation, Decision Support Systems 65 (2014), 95-104.

[37] L. Ding, P. Kolari, S. Ganjugunte, T. Finin and A. Joshi, Modeling and evaluating trust network inference, The 7th international workshop on trust in agent societies at AAMAS, New York, USA, 2004

[38] M. Lesani and N. Montazeri, Fuzzy trust aggregation and personalized trust inference in virtual social networks, Computational Intelligence 25(2) (2009), 51-83.
[39] G.R.P. French Jr, A formal theory of social power, Psychological review 63(3) (1956), 181.

[40] M. McPherson, L. Smith-Lovin and J.M. Cook, Birds of a feather: Homophily in social networks, Annual review of sociology (2001), 415-444.

[41] C.N. Ziegler and J. Golbeck, Investigating Interactions of Trust and Interest Similarity, Decision Support Systems 43 (2007), 460-475.

[42] J. Golbeck, Trust and Nuanced Profile Similarity in Online Social Networks, ACM Transactions on the Web 3(4) (2009), 12:1$12: 33$.

[43] G. Fernandez-Gago, I. Agudo and J. Lopez, Building Trust from Context Similarity Measures, Computer Standards \& Interfaces 36 (2014), 792-800.

[44] D. Gefen, Ecommerce: the role of familiarity and trust, Omega 28(5) (2000), 725-737.

[45] J. Zhang, A.A. Ghorbani and R. Cohen, A familiarity-based trust model for effective selection of sellers in multiagent ecommerce systems, International Journal of Information Security 6(5) (2007), 333-344.

[46] F. Hao, G. Min, M. Lin, C. Luo and L.T. Yang, MobiFuzzyTrust: an efficient fuzzy trust inference mechanism in mobile social networks, IEEE Transactions on Parallel and Distributed Systems 25(11) (2014), 2944-2955.

[47] R.L. Moreland and R.B. Zajonc, Exposure effects in person perception: Familiarity, similarity, and attraction, Journal of Experimental Social Psychology 18(5) (1982), 395-415.

[48] C.R. Goeldner and J.R.B. Ritchie, Tourism principles, practices, philosophies, New York: Wiley, 1990.

[49] C.R. Goeldner and J.R.B. Ritchie, Tourism principles, practices, philosophies, John Wiley \& Sons, 2007.

[50] A. María Munar, Tourist-created content: rethinking destination branding, International Journal of Culture, Tourism and Hospitality Research, 5(3) (2011), 291-305.

[51] P. De Meo, A. Nocera, G. Terracina and D. Ursino, Recommendation of similar users, resources and social networks in a Social Internetworking Scenario, Information Sciences 181(7) (2011), 1285-1305.

[52] Y.A. Kim and H.S. Song, Strategies for predicting local trust based on trust propagation in social networks, Knowledge-Based Systems 24(8) (2011), 1360-1371.

[53] Y.A. Kim, An enhanced trust propagation approach with expertise and homophily-based trust networks, Knowledge-Based Systems 82 (2015), 20-28.

[54] J. Tang, X. Hu and H. Liu, Social recommendation: a review, Social Network Analysis and Mining 3(4) (2013), 1113-1133.

[55] N.A. Christakis and J.H. Fowler, Connected: The surprising power of our social networks and how they shape our lives, Little, Brown, 2009.

[56] M. Jamali and M. Ester, Trustwalker: a random walk model for combining trust-based and item-based recommendation, Proceedings of the 15th ACM SIGKDD international conference on Knowledge discovery and data mining, 2009, 397-406.

[57] Y.M. Li, C.T. Wu and C.Y. Lai, A social recommender mechanism for e-commerce: Combining similarity, trust, and relationship, Decision Support Systems 55(3) (2013), 740-752.

[58] P. Massa and P. Avesani, Trust metrics on controversial users: Balancing between tyranny of the majority, International Journal on Semantic Web and Information Systems 3(1) (2007), 3964. 